\author{
Military Technical College \\ Kobry El-Kobbah, \\ Cairo, Egypt
}

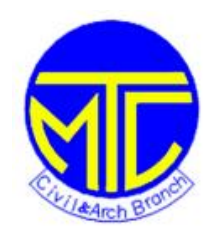

\section{$9^{\text {th }}$ International Conference on Civil and Architecture \\ Engineering \\ ICCAE-9-2012}

\title{
SENSORS USED IN STRUCTURAL HEALTH MONITORING
}

\author{
A. Abdelaal*, A. S. Mosallam**, and M. S. Amin***.
}

\begin{abstract}
Increased awareness of the economic and social effects of aging, deterioration and extreme events on civil infrastructure has been accompanied by recognition of the need for advanced structural health monitoring and damage detection tools. A number of new research projects have been funded to improve the damage detection methods including the use of innovative signal processing, new sensors, and control theory. Moreover, due to the dynamic nature of structures and their environment, creating and implementing the perfect sensors and their corresponding systems is quite difficult. This pursuit has been quite challenging for a multitude of reasons, which will be examined in this paper along with different types of sensors and preferences.
\end{abstract}

Keywords: structural health monitoring, damage detection, infrastructure, Sensors.

\section{INTRODUCTION:}

Civil infrastructure, which includes bridges and buildings, begin to deteriorate once they are built and used. Maintaining safe and reliable civil infrastructures for daily use is i mportant to the well being of all of us. Knowing the integrity of the structure in terms of its age and usage, and its level of safety to withstand infrequent but high forces such as overweight trucks, earthquakes, tornadoes, and hurricanes is important and necessary. The process of determining and tracking structural integrity and assessing the nature of damage in a structure is often referred to as health monitoring. Nowadays multiple structures worldwide are monitored with sensor arrays to monitor ambient vibration, strain and displacement among other parameters.

* Ph. D. student, Civil \& Environmental Eng. Dept., University of California , Irvine (UCI), U.S.A

** Professor, Civil \& Environmental Eng. Dept., University of California , Irvine (UCI ), U.S.A.

*** Egyptian Armed Forces. 
Sensors require high reliability, accuracy, high dynamic range, and immunity to electromagnetic and electrical interference, resistance to environmental conditions, such as low and high temperature, corrosion, and exposition to moisture, and severe shock and vibration. This requires ruggedness, durability and longevity of the sensors . [1]

In addition to the correct selection of sensors to match the previous conditions, they also have to be suitable for measurement of ke y parameters that influence the performance and health of the structural system. In addition to the structural considerations, sensors need to be chosen based on compatibility with the materials and the range and scope of measurements . [2]

\section{Overview}

As discussed earlier conventional sensor technology has a number of difficulties that must be overcome, the first being interference by electromagnetic fields. Generally advanced infrastructure systems house many devices that can cause strong electromagnetic inte rference. Also long cables can act as an antenna and pick up noise, generate ground loops and are susceptible to lightning strikes. Shielding the cables can prevent electric interference but not electromagnetic interference. Conventional sensors also do not always demonstrate the ruggedness, durability and longevity required by the hostile environmental conditions of the structures. Consistent research has shown that fiber optic sensors solve these set of problems.

\subsection{Optical fiber sensor}

Since the material of the optical fiber sensor is dielectric, it is immune to electromagnetic interference. Optical fiber sensors and cables are lightweight, carry light for excitation and sensed signal simultaneously through the same line and can be multiplexed. Electric sho rting due to moisture does not happen to fiber optics, so they don't have to be water -proofed. [1] Another great attribute of fiber optic sensors is that they have a distributed property meaning that they sense in two and three dimensional arrays. They are very resistant against a range of temperatures. Also because they are solid state they are capable of withstanding extremely high levels of vibration and shock. Also due to the continual exponential growth of the telecommunication market, the projected price of fiber optics is potentially low. By using an optical sensor in which the entire length of the fiber has the capability to detect damage, the number of sensors needed for the structure is greatly reduced. Fiber-optic Bragg grating sensors to monitor fatigue crack growth and delamination within hybrid laminates, embedded Bragg grating fiber -optic sensors can be used to monitor the strain in the layers near a fatigue crack. Fiber Bragg grating (FBG) sensors could be used for detecting transverse cracks in carbon fiber reinforced plastic (CFRP) cross -ply laminates. Because the FBG sensors are sensitive to nonuniform strain distribution along the length of the gratings, they have the potential for detecting damage that causes such strain in composite materials. 
The use of the plastic optical fibers is justified because the plastic optical fibers are much cheaper and easier to install than conventional silica fiber counterparts. Also, the plastic optical fibers have similar coefficients of thermal expansion and elasticity characteristics as the parent FRP structure, resulting in good bondage between the parent material and the optical fibers. However, the plastic optical fibers have much greater light attenuation than silica fibers, limiting their use to short distance transmission. Optical waveguide sensor with the capability to monitor strains up to 2,000 microstrain that are not affected by temperature, optical waveguide sensors are extremely sensitive and have the ability to measure large deformations . [3]

Fiber-optic Michelson interferometric sensors are used to detect failure in reinforced concrete beams. The beams were loaded in bending until fracture occurred. It has been found that the measurements from the fiber-optic sensors did not match the measure ments from the electrical strain gauges. However, the fiber-optic sensors were successful in detecting the failure of the beam. [3]

For long-term monitoring of structures SOFO deformation sensors are used. The SOFO optical fiber sensors, which are based on a Michelson interferometer, have been successful for measuring deformations that occur slowly over time. Each sensor consists of two optical fibers. One is installed in mechanical contact with the structure to be monitored, while the second, the reference fiber, is placed loose in a pipe for temperature compensation. Deformation of the structure is measured based on the difference of length changes between the two fibers. The lengths of the fibers are measured by interferometry . [2]

Although fiber optic sensors provide all these advantages over conventional sensors, fiber optic sensors weren't originally designed for use in civil infrastructures, and this posed some serious problems. They were originally designed for changes in light intensity, and phase de lay and interference in light patterns, so they were too sensitive in their measurements, which need a broad measurement range. They also measure the combined effects of changes such as temperature and strain, where in this situation we would only need str ain. The fiber's physical strength also put a limit on the maximum measured capacities of strain. So a newer innovation in fiber optic sensors was introduced. [3]

Changing the head to a transversely vibrating wire instead of an optical fiber, that senses quantities with no interference. This increased the sensors range and increases reliability and robustness. The wire's vibration frequency is detected by light reflected from the optical fiber. This wire is constantly oscillated by a series of laser pulses whose frequency is automatically and instantaneously adjusted to the resonant frequency of the wire, which changes according to wire tension. A PLL (Phase Locked Loop) circuit is used in order to ensure the exciting frequency matches the resonant frequency of the wire varying with the external force. This sensor can be 
modified to appropriately measure strain, acceleration and pressure variation. If a mass and damper are attached, it can serve as an accelerometer, if a large plate with appropriate stiffness is installed to one of the ends of the plate of the shell, it can be used as an anemometer, soil or water pressure sensor. Another advantage is the optical sensor is that a possibly a large number of these units can be combined to form a distributed senso r network, which has multiple sensor heads but uses only two light sources and only two fiber optic cables. Where one set of light source and optical fiber cable is for generating and transmitting the light pulse to excite the wires of all the sensor heads, which possibly all have different frequencies; while the other set of light source and optical fiber cable is for sending the detecting light to and back from the wires, where signals with different frequencies are multiplexed into one cable. Such a sens or network will reduce cost and eliminate cabling problems typically associated with conventional sensors . [1]

The fiber optic accelerometer is based on a novel integration of the Moire fringe phenomenon with fiber optics. The sensor head contains a pendu lum and a pair of parallel optical gratings panels; one fixed to the pendulum and the other to the sensor case. The relative displacement between the mass and the sensor case can be measured by "viewing" and counting the number of moiré fringes formed on the optical gratings using optical fibers. By properly designing the dynamic parameters of the pendulum, the relative displacement becomes proportional to the acceleration of the sensor case.

Compared with its conventional counterparts (electric sensors), this optical fiber accelerometer has total immunity to electromagnetic interference and lightning strikes due to the non-existence of electric signals or electric cables, unique safety in dangerous environments where electric sparks causes explosion con cerns, extremely high sensitivity, resolution, along with a large measuring range, particularly high performance in low frequencies, a small sensor head with a lightweight optical fiber cable, making it much easier to install on an existing structure, robustness against to environmental (temperature, moisture, etc.) changes due to the use of the well-understood and reliable measuring mechanism of the moiré phenomena, much lower cost than most of the optical fiber sensors due to its simple signal processing, a versatility to measure different dynamic quantities upon simple modification . [3]

\subsection{Microwave imaging technology}

Advanced composite materials are being used for seismic retrofit of reinforced concrete (RC) structures designed and constructed under older specifications, but the post-earthquake damage of the concrete structure covered by the composite jackets remains a significant concern. Addressing this is a microwave imaging technology for assessing the damage of the jacketed concrete structures. A unique microwave camera consisting of antenna arrays, together with special imaging algorithms and software packages, has recently been developed that can take a 3D microwave image with high accuracy and speed. This technology is applicable for quantitative 
condition assessment of concrete structures and pavements in addition to composite -retrofitted structures, thus significantly improving the effectiveness and efficiency of the current visual inspection-based maintenance and enhancing the safety, reliability, and longevity of concrete structures and pavements. [4]

\subsection{Video monitoring}

Video monitoring is also a tool in monitoring structural health. A pilot technology integration test bed used a PC-based data acquisition system with live internet connectivity that is deployed along a network camera that is used for continuous monitoring of a structure . [3] This particular configuration is used on highways. The system incorporates a large accelerometer array that synchronizes with a video monitoring system. All senso rs and data acquisition hardware are placed inside the bridge box girder. Accelerometers are positioned to measure vertical vibration on passing moving loads. The camera is of course used to relate passing vehicles to sensor output. Images are time stamped and sent to the image acquisition computer. This allows for accurate synchronization between the images and dynamic response of the sensors. All systems operate online via a high speed wireless internet network, allowing real -time data transmission. Image processing for this procedure includes background separation and vehicle detection. Background separation uses the Gaussian mixture model, and vehicle detection uses the background subtraction method. [5]

\subsection{Soil moisture sensors}

Soil moisture sensors measure the water content in soil. A soil moisture probe is made up of multiple soil moisture sensors. One common type of s oil moisture sensors in commercial use is a Frequency domain sensor such as a capacitance sensor. Another sensor, the neutron moisture gauge, utilizes the moderator properties of water for neutrons. Cheaper sensors -often for home use- are based on two electrodes measuring the resistance of the soil. Sometimes this s imply consists of two bare (galvanized) wires, but there are also probes with wires embedded in gypsum. Measuring soil moisture is important in agriculture to help farmers manage their irrigation systems more efficiently. Not only are farmers able to gener ally use less water to grow a crop, they are able to increase yields and the quality of the crop by better management of soil moisture during critical plant growth stages. Besides agriculture, there are many other disciplines using soil moisture sensors. Golf courses are now using sensors to increase the efficiencies of their irrigation systems to prevent over watering and leaching of fertilizers and other chemicals offsite. In urban and suburban areas, landscapes and residential lawns are using soil moisture sensors to interface with an irrigation controller. Connecting a soil moisture sensor to a simple irrigation clock will convert it into a "smart" irrigation controller that prevents an irrigation cycle when the soil is wet.[6] 
Frequency domain (FD) sensor is an instrument developed for measuring soil moisture content. The instrument has an oscillating circuit, the sensing part of the sensor is embedded in the soil, and the operating frequency will depend on the value of soil's dielectric constant.

There are two types of this sensors ; 1) Capacitance probe, or fringe capacitance sensor. Capacitance probes use capacitance to measure the dielectric permittivity of the soil. The volume of water in the total volume of soil most heavily influences the dielectric permittivity of the soil because the dielectric of water (80) is much greater than the other cons tituents of the soil (mineral

soil: 4, organic matter: 4, air: 1). Thus, when the amount of water changes in the soil, the probe will measure a change in capacitance (from the change in dielectric permittivity) that can be directly correlated with a change in water content. Circuitry inside some commercial probes changes the capacitance measurement into a proportional millivolt output . 2) Electrical impedance sensor, which consists of soil probes and using electrical impedance measurement. The most common configuration is based on the standing wave principle. The device comprises a $100 \mathrm{MHz}$ sinusoidal oscillator, a fixed impedance coaxial transmission line, and probe wires which is buried in the soil. The oscillator signal is propagated along the transmission line into the soil probe, and if the probe's impedance differs from that of the transmission line, a proportion of the incident signal is reflected back along the line towards the signal source . [6]

Compared to time domain reflectometer (TDR), FD sensors are cheaper to build and have a faster response time. However because of the complex electrical field around the probe, the sensor needs to be calibrated for different soil types. Some commercial sensors have been able to remove the soil type sensitivity by using a hig h frequency. [7]

\subsection{Sensors to measure moisture in Concrete}

Moisture has an important influence on the lifetime of concrete structures. In fact, water acts as a transport agent for damaging ions such as chloride, sulfate, carbonate, and ammonium. Corrosion of steel rebars is also an important factor in assessing the useful life of concrete structures. Fiber-optical moisture sensor had been developed to monitor the moisture content of concrete. Their measurement system consists of a plastic optical fiber, a light source, and a highresolution microspectrometer as well as a sensor. The sensor contains a polymer matrix that is attached directly to the concrete. An indicator dye is injected into that matrix, and the water content in the matrix affects the absorption spectra of the dye. As light from the source passes through the optical fibers to the sensor, the microspectrometer determines which wavelengths of light are absorbed by the polymer matrix/dye in the sensor. In this way, the moisture content of the concrete to which the sensor is directly attached can be determined. Without moisture, the sensor shows a peak wavelength in the absorption spectrum of $602 \mathrm{~nm}$. The peak wavelength is shifted about $40 \mathrm{~nm}$ to $562 \mathrm{~nm}$ when the water content in the sensor matrix is $28 \%$ by weight. The researchers confirm the long-term stability of the sensing system as well as the reproducibility of 
results when the moisture content of test specimens is wetted and dried in a cyclic manner. They also testify that the system can monit or the drying process of concrete. [3]

\subsection{MEMS Technology for Sensing Motion}

Micro Electro Mechanical Systems (MEMS) are miniature electromechanical sensor and actuator systems developed from the mature batch -fabricated processes of Very Large System Integration (VLSI) technologies. Advances in MEMS technologies have led to dramatic reductions in size, power consumption, and cost for wireless communications. Their small size allows them to be used in applications where conventional sensors and actuators woul $\mathrm{d}$ be intrusive. Because of the economies of scale achievable from the conventional chip manufacturing processes, they can be mass produced and copiously applied in a cost-effective manner. Furthermore, they consume very little power, on the order of $500 \mathrm{~W}$ to $40 \mathrm{~W}$. One example of MEMS wireless sensor modules, which incorporate a wireless telemetry system, on -board microprocessor, sensors, and batteries into a package about a cubic inch in size, is Courtesy of Berkeley Sensor \& Actuator Center, University of California: Berkeley. [3]

MEMS is an emerging technology through which miniature mechanical systems are built making use of the standard integrated circuits (IC) technologies on the same chip as the electronic circuitry. The main advantage of MEMS is that, because of the effectiveness in their fabrication process, they can perform measurements at relatively low cost and low power consumption. The field of MEMS has been developed since the end of the 1980s while, in comparison silicon-based sensors and actuators go back to the 1970s. Currently, MEMS are used for many applications such as communications (mobile phones), finding industrial, automotive industrial, medical, and security purposes. For dynamic monitoring of civil engineering structures, mechanical micro sensors (micro-accelerometers) are the most appropriate. [8]

It should, however, be noted that reliability and measurement accuracy are still problems that must be addressed for successful implementation of MEMS technologies to SHM. While MEMS sensing technologies are appropriate for local SHM applications, such as those that identify crack initiation, propagation, and corrosion, deploying a large number of MEMS devices over a large area in a cost-effective manner is a difficult problem. MEMS technologies can make this type of SHM system possible. A reduction in the signal processing load and power requirements is the main advantage of such a sensor. [3]

\subsection{Wireless sensor network (WSN)}

Another sensor has recently been introduced in sensor health monitoring; these are wireless sensors. They obviously have the distinct advantage of using no wiring. A large deployment of these wireless sensors is a very effective method of structural health monitoring. 
Besides offering a low cost relative to traditional cable based monitoring system, a wireless sensor network offers a distributed computing paradigm that allows sensor s to self-interrogate structural response data. Furthermore, wireless sensors utilize an implementation of the IEEE 802.15.4 which is standard for wireless networks to participate in scalable communication this makes it adaptable to network changes without requiring reprogramming. [3] This is very efficient for distributed sensor systems because it utilizes true ad -hoc peer-to-peer communication.

A wireless sensor network (WSN) consists of spatially distributed autonomous sensors to monitor physical or environmental conditions, such as temperature, sound, vibration, pressure, motion or pollutants and to cooperatively pass their data through the network to a main location. The more modern networks are bi-directional, also enabling control of sensor activity. The development of wireless sensor networks was motivated by military applications such as battlefield surveillance. Today such networks are used in many industrial and consumer applications, such as industrial process monitoring and control, machine health monitoring, etc. In computer science and telecommunications, wireless sensor networks are an active research area with numerous workshops and conferences arranged each year. The WS N is built of "nodes" from a few to several hundreds or even thousands, where each node is connected to one (or sometimes several) sensors. Each such sensor network node has typically several parts: a radio transceiver with an internal antenna or connection to an external antenna, a microcontroller, an electronic circuit for interfacing with the sensors and an energy source, usually a battery or an embedded form of energy harvesting. A sensor node might vary in size from that of a shoebox down to the size of a grain of dust, although functioning "motes" of genuine microscopic dimensions have yet to be created. The cost of sensor nodes is similarly variable, ranging from a few to hundreds of dollars, depending on the complexity of the individual sensor nodes. Size and cost constraints on sensor nodes result in corresponding constraints on resources such as energy, memory, computational speed, and communications bandwidth. The topology of the WSNs can vary from a simple star network to an advanced multi-hop wireless mesh network. The propagation technique between the hops of the network can be routing or flooding . [9]

Area monitoring is a common application of WSNs. In area monitoring, the WSN is deployed over a region where some phenomenon is to be monitored. As a military example, with the use of sensors they can detect enemy intrusion. A civilian example is the geo -fencing of gas or oil pipelines. When the sensors detect the event being monitored (heat, pressure), the e vent is reported to one of the base stations, which then takes appropriate action (e.g., send a message on the internet or to a satellite). Similarly, wireless sensor networks can use a range of sensors to detect the presence of vehicles ranging from motor cycles to train cars. For water monitoring, there are many opportunities for using wireless sensor networks within the water industries. One example is to detect the moisture and water levels in the soil. Facilities not wired for power or data transmission can be monitored using industrial wireless $\mathrm{I} / \mathrm{O}$ devices and sensors powered 
using solar panels or battery packs. Also, they are used in pollution control boards. For structural monitoring, wireless sensors can be used to monitor the movement within buildin gs and infrastructure such as bridges, flyovers, embankments, tunnels etc. This enabling engineering practices to monitor assets remotely without the need for costly site visits, as well as having the advantage of daily data, whereas traditionally this da ta was collected weekly or monthly, using physical site visits, involving either road or rail closure in some cases. It is also far more accurate than any visual inspection that would be carried out . [10]

Several standards are currently either ratified or under development for wireless sensor networks. There are a number of different standardizations in the field of WSNs. The IEEE focuses on the physical and MAC layers; the Internet Engineering Task Force works on layers 3 and above. In addition to these, there is the International Society of Automation which provides vertical solutions, covering all protocol layers. Finally, there are also several non -standard, proprietary mechanisms and specifications. Standards are used far less in WSNs than in other computing systems which make most systems incapable of direct communication between different systems. However predominant standards commonly used in WSN communications include: Wireless HART, ISA100, IEEE 1451, and ZigBee/802.15.4. [11]

The main features of a WSN include: The ability to cope with node failures, the mobility of nodes, dynamic network topology, communication failures, heterogen eity of nodes, scalability to large scale of deployment, the ability to withstand harsh environmental conditions, ease of use, unattended operation, and power consumption. Although power consumption constrains for nodes using batteries or energy harvesting is limited. Wireless sensors are usually powered by their own local battery. Since this power supply is limited relative to wire based systems, they should operate on low power circuits and have well programmed power efficient algorithms . [12]

For peer-to-peer programming the IEEE 802.15.4 (LR-WPAN) is good. The features of a LR-WPAN utilizing this standard are wireless connectivity over short ranges, low cost to build, easy installation, reliable data transfer, and efficient use of battery power. The LR-WPAN protocol is concentrated in the bottom two layers of the communication protocol stack, namely the PHY (physical access) layer and the MAC (medium access control) layers. The PHY layer is intended to coordinate the physical radio to modulate and demodu late data upon carrier radio frequencies. The MAC layer offer standard packet structures for data transmission as well as procedural rules for autonomous users to share the common bandwidth . [13]

Sensor nodes can be imagined as small computers, extremely b asic in terms of their interfaces and their components. They usually consist of a processing unit with limited computational power and limited memory, sensors or MEMS (including specific conditioning circuitry), a communication device (usually radio transceivers or alternatively optical), and a 
power source usually in the form of a battery. Other possible inclusions are energy harvesting modules, secondary ASICs, and possibly secondary communication devices (e.g. RS-232 or USB). [11]

The base stations are one or more components of the WSN with much more computational, energy and communication resources. They act as a gateway between sensor nodes and the end user as they typically forward data from the WSN on to a s erver. Other special components in routing based networks are routers, designed to compute, calculate and distribute the routing tables. Many techniques are used to connect to the outside world including mobile phone networks, satellite phones, radio modem s, long-range Wi-Fi links etc. Many base stations are ARM-based (Advanced RISC Machine) running a form of Embedded Linux. [14]

If a centralized architecture is used in a sensor network and the central node fails, then the entire network will collapse, however the reliability of the sensor network can be increased by using distributed control architecture. Distributed control is used in WSNs for the following reasons: Sensor nodes are prone to failure, for better collection of data, and to provide nodes with backup in case of failure of the central node. There is also no centralized body to allocate the resources and they have to be self-organized. The data gathered from wireless sensor networks is usually saved in the form of numerical data in a central base station. Additionally, the Open Geospatial Consortium (OGC) is specifying standards for interoperability interfaces and metadata encodings that enable real time integration of heterogen eous sensor webs into the Internet, allowing any individual to monitor or control Wireless Sensor Networks through a Web Browser. [15]

To reduce communication costs some algorithms remove or reduce nodes redundant sensor information and avoid forwarding da ta that is of no use. As nodes can inspect the data they forward they can measure averages or directionality. For example, in sensing and monitoring applications, it is generally the case that neighboring sensor nodes monitoring an environmental feature typically register similar values. This kind of data redundancy due to the spatial correlation between sensor observations inspires the techniques for in -network data aggregation and mining. By taking into account the spatial correlation between data sampled by different sensors, a wide class of specialized algorithms can be used to implement more efficient spatial data mining algorithms, as well as more efficient routing in the network. [14]

In wireless sensor networks, information fusion, also called data $\mathrm{f}$ usion, has been developed for processing sensor data by filtering, aggregating, and making inferences about the gathered data. Information fusion deals with the combination of multiple sources to obtain improved information: cheaper, greater quality or gre ater relevance. Within the wireless sensor networks domain, simple aggregation techniques such as maximum, minimum, and average, have been developed for reducing the overall data traffic to save energy. [12] 
Visual sensors are much more costly and time con suming than wireless sensors. Traditional wire based systems are also much more expensive than wireless systems. In the year 2000, an inspection of the Golden Gate Bridge wired system cost \$226,900 [7]. A large part of this cost is due to the wires needed to cover the entire bridge. Wireless sensors are economic and work autonomously. Another special attribute of wireless sensors is their ability of collocation of computing power with the sensor, meaning that data can be analyzed and processed in the sensor , rather than it being used as a raw instrumentation tool. Several custom -based algorithms have been designed for the sensors for damage detection and system identification . [16]

The best damage detection wireless systems are those that involve peer to pe er communication and distributed in network computation. As long as it is taken into consideration that the optimal spacing between sensors must be met. Also communication between the sensors should be kept minimal as radio waves because this is the greate st consumer of power in the sensor. The wireless sensor network also should be scalable so that it is not limited to size or number of wireless nodes. A lot of research is geared towards improving circuit environment to improve fidelity, using low powered radio and a better communication protocol for accurate time synchronization and network scalability. [15]

\section{CONCLUSION:}

Various sensors are used to monitor structural health as examined in this paper. The most efficient sensors for structural health monit oring are Moiré fringe-based fringe optical fiber sensors and wireless sensors. Moiré fringe-based fringe optical fiber sensors are preferred due to their robustness, reliability, and ability to adapt to dynamic structures. Wireless sensors are used due to their economic feasibility, and increased accuracy via distributed networking. MEMS sensing technologies have the advantage of being able to reduce the signal processing load and power requirements. While MEMS are appropriate for local SHM applications, reliability and measurement accuracy are still problems that must be addressed for successful implementation of MEMS technologies to SHM. In my opinion, the optimal use of network for SHM would be the integration of both Moiré fringe-based optical fiber sensors with a wireless system in order to get the advantages of both systems.

\section{REFERENCES:}

[1] Feng, M. Q, Suzuki, H, and Yokoi, I (1995). "Development of Optical Sensing Systems for Smart Civil Infrastructure”, Journal of Smart Materials and Structures, No. 4, Vol. 1995, pp. A114-A120

[2] Liang Y, Tennant A, Jia H, Xiong X, Ansari F (2005) "Implementation of Long Gauge Fiber Optic Sensor Arrays in Civil Structures" Chapter VII. Fiber Optic Sensors Principles 
[3] Los Alamos National Library 2003 “A Review of Structural Health Monitoring Literature: 19962001” Los Alamos National Laboratory Report LA 13976-MS. Los Alamos, NM USA

[4] M. Rivas Lopez, O. Yu. Sergiyenko, V.V. Tyrsa, W. Hernandez Perdomo, L.F. Devia Cruz, D. Hernandez Balbuena, L.P. Burtseva, and J.I. Nieto Hipolito "Optoelectronic Method for Structural Health Monitoring” Structural Health Monitoring, March 2010; vol. 9, 2: pp. 105-120., first published onSeptember 24, 2009

[5] Ricardo Zaurin and F. Necati Catbas "Structural health monitoring using videostream, influence lines, and statistical analysis" Structural Health Monitoring, May 2011; vol. 10, 3: pp. 309-332., first published onJune 28, 2010

[6] Gaskin G.J., Miller J.D. 1996. Measurement of soil water content using a simplified impedanc e measuring technique. Journal of Agricultural Engineering Research 63, 153-160.

[7] Campbell C.S., Campbell G.S., Cobos D.R.2004. "Response of Low Cost Dielectric Moisture Sensor to Temperature Variation”. Eos Trans. AGU, 85(17), Jt. Assem. Suppl. Abstract NS44A-05.

[8] Luis F Ramos, Rafael Aguilar, and Paulo B Lourenço “Operational modal analysis of historical constructions using commercial wireless platforms" Structural Health Monitoring, September 2011; vol. 10, 5: pp. 511-521. First published on November 18, 2010.

[9] Dargie, W. and Poellabauer, C., "Fundamentals of wireless sensor networks: theory and practice", John Wiley and Sons, 2010 ISBN 978-0-470-99765-9, pp. 168-183, 191-192.

[10] Lynch J, A Sundarajan, H Law, 2005, "Embedding Damage Detection Algorithms in a Wireless Sensing Unit for Attainment of Operational Power Efficiency" Smart Mater. Structure, 13(4):800-810

[11] Soro S., Heinzelman W.: A Survey of Visual Sensor Networks, Advances in Multimedia, vol. 2009, Article ID 640386, 21 pages, 2009. doi:10.1155/2009/640386.

[12] Eduardo F. Nakamura, Antonio A. F. Loureiro, Alejandro C. Frery. Information fusion for wireless sensor networks: Methods, models, and classifications, ACM Computing Surveys, Volume 39, Issue 3, Article 9, September 2007.

[13] IEEE Computer Society. 2003 IEEE Standard 802.15.4, The Institute of Electrical and Electronics Engineering, Inc. 3 Park Avenue, New York, NY 10016-5997, USA

[14] Ma, Y.; Guo, Y.; Tian, X.; Ghanem, M. (2011). "Distributed Clustering-Based Aggregation Algorithm for Spatial Correlated Sensor Networks". IEEE Sensors Journal 11 (3): 641. doi: $10.1109 /$ JSEN.2010.2056916

[15] Andrew S, Jung D, Lynch J, Wang Y, Shi D,Flynn M "Design of a Wireless Sensor for Scalable Distributed In-Network Computation in a Structural Health Monitoring Syst em" Proceedings of the 5th International Workshop on Structural Health Monitoring 2005

[16] Frasier M, Elgamal A, He X, Conte J (2010) "Sensor Network for Structural Health Monitoring of a Highway Bridge" Journal of Computing in Civil Engineering, Vol. 24, No. 1, January/February 2010, pp. 11-24 